Rev. Biol. Trop., 48(2/3): 413-424, 2000

www.ucr.ac.cr www.ots.ac.cr www.ots.duke.edu

\title{
Los peces del arrecife de Cabo Pulmo, Golfo de California, México: Lista sistemática y aspectos de abundancia y biogeografía
}

\author{
Antonio Villarreal-Cavazos, Héctor Reyes-Bonilla*, Benito Bermúdez-Almada \& Oscar Arizpe- \\ Covarrubias. \\ Universidad Autónoma de Baja California Sur. Departamento de Biología Marina. Apartado postal 19-B, CP 23080. \\ La Paz, B.C.S., México. Fax (112) 1-28-01. \\ * Correspondencia. Dirección actual: University of Miami. Rosenstiel School of Marine and Atmospheric Science. \\ Division of Marine Biology and Fisheries. 4600 Rickenbacker Causeway, Miami, FL 33149. EEUU. Correo elec- \\ trónico: hreyes@ rsmas.miami.edu
}

Recibido: 9-III-1999. Corregido 22-XI-1999. Aceptado 1-XII-1999.

\begin{abstract}
The Cabo Pulmo reef is the most important coral formation of the Gulf of California; however, its ichthyological fauna has been poorly studied. To produce a systematic list with data on relative abundance and frequency, and biogeographical affinities, we relied on visual census, field observations, analysis of commercial and sport fisheries (from 1986 to 1998), and the literature. A total of 236 species have been recorded at Cabo Pulmo (155 genera and 60 families). This number doubles previous compilations and represents $65.1 \%$ of all reef fishes known from the Gulf of California, and about 35\% of its entire shallow-water fishes. Of the total species number, $68.3 \%$ are from the Panamic Province, 11.0\% Indo Pacific colonizers and the same percentage gulf endemics, $7.6 \%$ are circumtropical, $1.7 \%$ Atlantic and $0.5 \%$ cosmopolitan; none are endemic to the reef. The most abundant taxa are the labrids Thalassoma lucasanum, $T$. grammaticum and the pomacentrid Chromis atrilobata. Only eleven species (4.7\% of total) appeared in $75 \%$ to $100 \%$ of census, and 36 (15.3\% of total) had high levels of both abundance and frequency, evidencing that the community is dominated by few taxa. Local species richness exceeds the number reported for most rocky or coral reefs of the Pacific coast of México, and indicates that Cabo Pulmo is a key area in the gulf and the entire Mexican Pacific, from the ichthyological point of view.
\end{abstract}

Keywords: Systematic list, abundance, biogeography, fishes, Cabo Pulmo reef, Gulf of California.

El arrecife coralino de Cabo Pulmo, situado en los $23.5^{\circ} \mathrm{N}$ y $109.5^{\circ} \mathrm{W}$, representa un tipo de ecosistema único en el Golfo de California y posee una notable riqueza de especies marinas; por estas razones, el gobierno de México lo declaró Parque Marino en 1995 (Reyes-Bonilla 1997). La zona ha sido objeto de variados estudios, y han aparecido listados de algunos grupos importantes de algas e invertebrados del sistema (Brusca \& Thomson 1975, Anaya-Reyna \& Riosmena-Rodríguez 1996, Reyes-Bonilla et al. 1997, Cintra-Buenrostro et al. 1998, Baynes 1999, García-Madrigal \& Bastida-Zavala 1999,
García-Madrigal en prensa). No obstante, la información sobre la composición ictiológica del arrecife es relativamente pobre. Aunque varios autores han hecho mención aislada de la presencia de algunas especies observadas o recolectadas en el arrecife o en aguas cercanas (e.g. Steinbeck \& Ricketts 1941, Thomson et al. 1979, Montgomery 1980a, b, de la Cruz-Agüero 1997), Brusca \& Thomson (1975) llevaron a cabo el único estudio que se refiere específicamente a las especies de peces de la zona, donde se presentó un listado de 108 especies, y se hizo 
mención de su abundancia relativa y otras características biológicas.

Para ayudar a llenar este vacío en el conocimiento, el objetivo del presente trabajo fue llevar a cabo una revisión de campo y literatura con el fin de conocer el elenco sistemático de los peces del arrecife de Cabo Pulmo. La información es importante debido a que los peces juegan un papel clave dentro de la red trófica del sistema coralino (Villarreal-Cavazos 1988), además de que representan los animales más conspicuos en la zona y su presencia eleva su relevancia como sitio de recreación (Reyes-Bonilla 1997).

\section{MATERIALES Y MÉTODOS}

Para la elaboración del listado de especies de peces el arrecife de Cabo Pulmo se llevaron a cabo labores de campo y búsquedas extensivas de literatura. En el primer caso, se hicieron más de 50 visitas a la localidad entre los años de 1986 y 1998 (sin seguir alguna periodicidad particular), durante las que se condujeron más de 200 buceos, cada uno de entre 30 y $60 \mathrm{mi}-$ nutos. A lo largo de este tiempo se fueron recopilando los registros de las especies observadas, pero no se llevaron a cabo recolectas ya que el objetivo de tales trabajos era el de apoyar la propuesta de conservación del área y la instalación y aplicación de un plan de manejo. En adición, en 1987, 1992, 1993, 1997 y 1998 se realizaron censos visuales de organismos utilizando la técnica del cilindro estacionario (Bohnsack \& Bannerot 1986) modificada por Villarreal-Cavazos (1988). Básicamente, se hicieron conteos de los peces que aparecieron dentro de un cilindro de observación de $7.5 \mathrm{~m}$ de radio, el cual cubría desde el fondo hasta la superficie ( $176.7 \mathrm{~m}^{2}$ totales de área de base por muestreo). El tiempo de observación de cada censo fue de $15 \mathrm{~min}$, usándose los primeros 7.5 min en anotar las especies vistas en el área de muestreo, y los restantes en contar el número de individuos de cada una; si aparecían nuevos táxones en la zona de censo luego del tiempo límite inicial, éstos no eran considerados. Para- lelamente, un segundo buzo enumeraba las especies y sus abundancias en la zona de rocas, arena o coral del fondo, ya que en el arrecife aparecen muchas especies crípticas o demersales. La metodología empleada presenta algunos inconvenientes para estimar la abundancia de especies territorialistas, pero es una de las mejores a aplicar cuando lo importante es determinar riquezas específicas (Sale 1997). Los censos $(\mathrm{N}=98 ; 21$ en 1987, 10 en 1992, 15 en 1993,10 en 1997 y 42 en 1998) se llevaron a cabo en todas las zonas del arrecife, y fueron conducidos hasta una profundidad máxima de $30 \mathrm{~m}$, pero en su mayoría estuvieron circuunscritos entre 3 y $10 \mathrm{~m}$, ya que en este intervalo se presenta la zona coralina principal (ReyesBonilla 1993). Las referencias para la identificación de las especies fueron múltiples y se presentan en Villlarreal-Cavazos (1988). De los trabajos más recientes consultados, pueden mencionarse los de Allen \& Robertson (1994) y Fischer et al. (1995).

Paralelamente a las labores de campo, durante los años previos a la declaratoria de protección del área se analizó la composición específica de la captura comercial llevada a cabo en el sitio, así como la de la pesquería deportiva, y se formó una lista nominal de las especies registradas en la literatura publicada sobre Cabo Pulmo. El elenco total de especies fue depurado para corregir sinonimias y confirmar la posición taxonómica de los táxones con base en los trabajos de Allen \& Robertson (1994), Fischer et al. (1995) y de la CruzAgüero (1997). A nivel de grupos mayores, se siguió la clasificación de Nelson (1994).

Una vez con la lista definitiva de especies y utilizando exclusivamente los datos de abundancia total de los peces censados en el arrecife entre 1987 y 1998, se categorizó cada taxon de manera relativa en tres niveles: Abundante (presentó más del 5\% de la abundancia total registrada); Común (especies cuyo número alcanzó entre el $1 \%$ y 5\% del total); Escaso (del 0.1 al $1 \%)$.

Complementariamente, la frecuencia de registro de las especies en los censos realizados también se clasificó en tres tipos: cuando 
las especies aparecieron del $75 \%$ al $100 \%$ de los muestreos, del $50 \%$ al $75 \%$ o bien, del $25 \%$ al $50 \%$. En todos los casos, aquellas que tuvieron cifras menores a las citadas, no recibieron calificación. Los niveles de abundancia y ocurrencia presentados en el trabajo están tomados en referencia a la totalidad del tiempo de estudio, por lo que no reflejan los cambios que algunas especies han sufrido (ReyesBonilla et al. en prep.), o las condiciones de años particulares.

\section{RESULTADOS}

En el arrecife de Cabo Pulmo se ha regisudo un total de 236 especies de peces, pertenecientes a 155 géneros y 60 familias (Cuadro 1). La mayoría (161 especies; $68.3 \%$ del total) habitan en la Provincia Panámica (sensu Galván-Magaña et al. 1996), aunque también hay una proporción alta de especies del Indo Pacífico y endémicas del Golfo de California (26 táxones cada una; $11 \%$ del total en ambos casos), circuntropicales (18; 7.6\% de la fauna), Atlánticas (4 táxones; $1.7 \%$ del total) y cosmopolitas $(1 ; 0.5 \%)$. No existen especies endémicas del arrecife.

Desde el punto de vista de la abundancia, las especies principales en el arrecife durante los años de observación fueron Thalassoma lucasanum (Gill, 1863), T. grammaticum Gilbert, 1890 y Chromis atrilobata Gill, 1862 (Cuadro 1). Considerando la frecuencia de registro, las dos primeras especies junto con otras nueve ( $4.7 \%$ del total) formaron el grupo principal y aparecieron del $75 \%$ al $100 \%$ de los censos. Las especies dominantes (aquellas que ocuparon altos niveles tanto de abundancia como de frecuencia) fueron sólo $36(15.3 \%$ del total), lo que indica que aún cuando la riqueza total es alta, el funcionamiento de la comunidad debe estar dirigido por pocos táxones.

\section{CUADRO 1}

Listado sistemático, abundancia y frecuencia relativa, y afinidad biogeográfica de los peces del arrecife de Cabo Pulmo, México.

TABLE 1

Systematic list, relative abundance and frequency, and biogeographical affinity of the fishes of Cabo Pulmo reef, México.

Phylum Chordata

Clase Chondrichthyes

Orden Orectolobiformes

Familia Rhincodontidae

Rhincodon typus Smith, 1828 [CT] [OO, *]

Orden Lamniformes

Familia Alopiidae

Alopias vulpinus (Bonnaterre, 1788) [CT] [HQ]

Orden Carcharhiniformes

Familia Carcharhinidae

Carcharhinus limbatus (Valenciennes, 1839) [CT] [BT, OO]

Negaprion brevirostris (Poey, 1868) [ANF] [HQ]

Orden Rajiformes

Familia Narcinidae

Diplobatis ommata (Jordan y Gilbert, 1890) [EP] [OO, *]

Narcine entemedor Jordan y Starks, 1895 [EP] [*]

Familia Dasyatidae

Dasyatis brevis (Garman, 1880) [EP] [*]

Dasyatis longus (Garman, 1880) [EP] [*] 
Familia Urolophidae

Urobatis concentricus Osburn y Nichols, 1916 [EP] [BT, OO, *]

Urobatis maculatus Garman, 1913 [END] [DC, *]

Familia Myliobatidae

Aetobatus narinari (Euphrasen, 1790) [COS] [BT, *]

Myliobatis longirostris Applegate y Fitch, 1964 [END] [*]

Familia Mobulidae

Manta birostris (Donndorff, 1798) [CT] [SR, *]

Clase Osteichthyes

Orden Anguilliformes

Familia Muraenidae

Anarchias galapagensis (Seale, 1940) [EP] [BT, OO]

[1] Echidna nebulosa (Ahl, 1789) [IP] [OO, *]

Echidna nocturna (Cope, 1872) [EP] [OO, *]

Enchelycore octaviana (Myers y Wade, 1941) [EP] [BT, OO]

[1] Gymnomuraena zebra (Shaw, 1797) [IP] [BT, TH, DC, OO, *]

[E] [1] Gymnothorax castaneus (Jordan y Gilbert, 1882) [EP] [BT, TH, OO, *]

Gymnothorax panamensis (Steindachner, 1876) [EP] [BT, OO, *]

[1] Muraena lentiginosa Jenyns, 1892 [EP] [BT, OO, *]

Uropterygius macrocephalus (Bleeker, 1865) [IP] [OO]

Uropterygius polystictus Myers y Wade, 1941 [IP] [OO]

Uropterygius tigrinus Girard, 1859 [EP] [*]

Familia Ophichthidae

Callechelys eristigma Mc.Cosker y Rosenblatt, 1972 [IP] [OO]

Ichthyapus selachops (Jordan y Gilbert, 1882) [EP] [OO]

Myrichtys maculosus (Cuvier, 1817) [IP] [OO]

Paraletharchus pacificus (Osburn y Nichols, 1916) [EP] [OO]

Phaenomonas pinnata Myers y Wade, 1941 [IP] [OO]

Quassiremus nothochir (Gilbert, 1890) [EP] [OO]

Scytalichthys miurus (Jordan y Gilbert, 1882) [IP] [OO]

Familia Congridae

Gorgasia punctata Meek y Hildebrand, 1923 [EP] [TH, OO, *]

Heteroconger digueti (Pellegrin, 1923) [EP] [OO, *]

Orden Clupeiformes

Familia Clupeidae

Harengula thrissina (Jordan y Gilbert, 1882) [EP] [BT, DC, OO, *]

Orden Aulopiformes

Familia Synodontidae

Synodus lacertinus Gilbert, 1890 [EP] [OO]

Orden Ophidiiformes

Familia Ophidiidae

Ophidion iris Breder, 1926 [END] [OO]

Familia Bythitidae

Oligopus diagrammus (Heller y Snodgras, 1903) [EP] [OO]

Orden Lophiiformes

Familia Antennariidae

Antennarius sanguineus Gill, 1863 [EP] [BT, OO]

Antennatus strigatus (Gill, 1863) [EP] [OO] 
Orden Gobiesociformes

Familia Gobiesocidae

Arcos erythrops (Jordan y Gilbert, 1882) [EP] [BT, OO]

Gobiesox adustus Jordan y Gilbert, 1882 [EP] [BT, OO]

Gobiesox pinniger Gilbert, 1890 [END] [BT, OO]

Tomicodon boehlkei Briggs, 1955 [END] [BT, OO]

Tomicodon eos (Jordan y Gilbert, 1882) [EP] [BT, OO]

Tomicodon myersi Briggs, 1955 [EP] [OO]

Tomicodon zebra (Jordan y Gilbert, 1882) [EP] [DC, OO]

Orden Beloniformes

Familia Hemiramphidae

Hemiramphus saltator Gilbert y Starks, 1904 [EP] [*]

Familia Belonidae

Platybelone argulus pterura (Osburn y Nichols, 1916) [CT] [OO]

Tylosurus pacificus (Steindachner, 1876) [EP] [BT?, OO, *]

Orden Atheriniformes

Familia Atherinidae

Eurystole eriarcha (Jordan y Nichols, 1916) [EP] [OO]

Orden Beryciformes

Familia Holocentridae

Myripristis leiognathos Valenciennes, 1846 [EP] [BT, OO, *]

[E] [1] Sargocentron suborbitalis (Gill, 1864) [EP] [BT, DC, OO, *]

Orden Syngnathiformes

Familia Fistularidae

[E] [1] Fistularia commersonii Ruppel, 1835 [IP] [OO, *]

Familia Syngnathidae

Cosmocampus arctus (Jenkins y Evermann, 1889) [EP] [OO]

Doryrhamphus excisus Kaup, 1856 [IP] [BT, OO]

Hippocampus ingens Girard, 1858 [EP] [*]

Orden Scorpaeniformes

Familia Scorpaenidae

Scorpaena plumieri mystes (Jordan y Starks, 1895) [EP] [BT, OO, *]

Scorpaenodes xyris (Jordan y Gilbert, 1882) [EP] [BT, OO, *]

Familia Triglidae

Bellator loxias Jordan en Gilbert, 1896 [EP] [OO]

Orden Perciformes

Familia Serranidae

Alphestes immaculatus Breder, 1936 [EP] [OO, *]

Dermatolepis dermatolepis (Boulenger, 1895) [EP] [*]

Epinephelus acanthistius (Gilbert, 1892) [EP] [HQ]

Epinephelus analogus Gill, 1864 [EP] [HQ, *]

Epinephelus itajara (Lichstenstein, 1822) [ANF] [BT, OO, *]

[E] [3] Epinephelus labriformis (Jenyns, 1843) [EP] [BT, DC, OO, *]

[E] [1] Epinephelus panamensis (Steindachner, 1876) [EP] [OO, *]

Hemanthias peruanus (Steindachner, 1874) [EP] [DC]

Mycteroperca jordani (Jenkins y Evermann, 1889) [EP] [*]

Mycteroperca prionura Rosenblatt y Zahuranec, 1967 [END] [*]

[E] [1] Mycteroperca rosacea (Streets, 1877) [EP] [BT, OO, *]

Mycteroperca xenarcha Jordan, 1888 [EP] [*]

[1] Paranthias colonus Valenciennes, 1855 [EP] [OO, *]

Serranus psittacinus Valenciennes, 1855 [EP] [OO, *]

Familia Grammistidae

Pseudogramma thaumasium (Gilbert, 1900) [EP] [BT, OO]

Rypticus bicolor Valenciennes, 1846 [EP] [BT, OO, *]

Rypticus nigripinnis Gill, 1861 [EP] [OO] 
Familia Priacanthidae

Cookeolus japonicus (Cuvier, 1829) [CT] [DC]

Heteropriacanthus cruentatus (Lacepede, 1801) [CT] [OO, *]

Familia Apogonidae

Apogon retrosella Gill, 1863 [EP] [BT, OO, *]

Familia Branchiostegidae

Caulolatilus affinis Gill, 1865 [EP] [DC, *]

Caulolatilus princeps (Jenyns, 1842) [EP] [DC]

Familia Carangidae

Alectis ciliaris (Bloch, 1788) [CT] [OO]

Caranx caballus Gunther, 1868 [EP] [BT, DC, *]

Caranx lugubris Poey, 1860 [CT] [OO, *]

Caranx otrynter (Jordan y Gilbert, 1883) [EP] [OO]

Gnathanodon speciosus Forskaal, 1775 [IP] [BT, OO]

Selar crumenophthalmus (Block, 1793) [CT] [HQ]

Selene brevoorti (Gill, 1863) [EP] [BT]

[E]

Trachinotus rhodopus Gill, 1863 [EP] [BT, *]

Familia Coryphaenidae

Coryphaena hippurus Linnaeus, 1758 [CT] [*]

Familia Lutjanidae

Hoplopagrus guntheri Gill, 1862 [EP] [BT, HQ, OO, *]

[1] Lutjanus argentiventris (Peters, 1869) [EP] [BT, OO, *]

Lutjanus colorado Jordan y Gilbert, 1882 [EP] [*]

Lutjanus guttatus (Steindachner, 1869) [EP] [HQ]

Lutjanus novemfasciatus Gill, 1862 [EP] [BT, OO, *]

[C] [2] Lutjanus viridis (Valenciennes, 1845) [EP] [BT, OO, *]

Familia Haemulidae

[E] Anisotremus davidsonii (Steindachner, 1875) [EP] [*]

[C] Anisotremus interruptus (Gill, 1862 [EP] [BT, OO, *]

[E] [1] Anisotremus taeniatus (Gill, 1861) [EP] [BT, *]

[E] Haemulon flaviguttatum Gill, 1863 [EP] [*]

[E] [1] Haemulon sexfasciatum Gill, 1863 [EP] [BT, DC, *] Microlepidotus inornatus Gill, 1862 [EP] [BT, OO, *]

Familia Sparidae

Calamus brachysomus (Lockington, 1880) [EP] [DC, *]

Familia Sciaenidae

Pareques viola (Gilbert en Jordan y Evermann, 1898) [EP] [BT, OO, *]

Umbrina dorsalis Gill, 1862 [EP] [DC]

Umbrina roncador Jordan y Gilbert, 1881 [EP] [BT, OO]

Umbrina xanti Gill, 1862 [EP] [DC]

Familia Mullidae

[E] [1] Mulloidichthys dentatus (Gill, 1863) [EP] [BT, OO, *]

Familia Kyphosidae

[E] Girella simplicidens Osburn y Nichols, 1916 [END] [*]

Hermosilla azurea Jenkins y Evermann, 1889 [EP] [BT, OO]

[E] Kyphosus analogus (Gill, 1863) [EP] [*]

[E] [1] Kyphosus elegans (Peters, 1869 [EP] [BT, HQ, OO, *]

Familia Ephippidae

Chaetodipterus zonatus (Girard, 1858) [EP] [BT, OO, *] 
Familia Chaetodontidae

Chaetodon falcifer Hubbs y Rechnitzer, 1958 [EP] [*]

Chaetodon humeralis Gunther, 1860 [EP] [OO, *]

Forcipiger flavissimus Jordan y Mc.Gregor, 1898 [IP] [*]

[C] [3] Johnrandallia nigrirostris (Gill, 1862) [EP] [BT, OO, *]

Familia Pomacanthidae

Holacanthus clarionensis Gilbert, 1890 [EP] [TH, DC, OO, *]

[C] [3] Holacanthus passer Valenciennes en Cuvier y Valenciennes, 1846 [EP] [BT, DC, OO, *]

[E] Pomacanthus zonipectus (Gill, 1862) [EP] [BT, OO, *]

Familia Pomacentridae

Abudefduf declivifrons (Gill, 1862) [EP] [BT, OO, *]

[C] [2] Abudefduf troschelii (Gill, 1862) [EP] [BT, OO, *]

[E] Chromis alta Greenfield y Woods, 1980 [EP] [*]

[A] [1] Chromis atrilobata Gill, 1862 [EP] [BT, OO, *]

Chromis limbaughi Greenfield y Woods, 1980 [END] [*]

Microspathodon bairdii (Gill, 1862) [EP] [BT, M, OO, *]

[C] [2] Microspathodon dorsalis (Gill, 1862) [EP] [BT, M, OO, *]

Stegastes acapulcoensis (Fowler, 1944) [EP] [TH,

[E] Stegastes flavilatus (Gill, 1863) [EP] [BT, OO, *]

Stegastes leucorus (Gilbert, 1892) [EP] [*]

[C] [3] Stegastes rectifraenum (Gill, 1863) [END] [BT, OO, *]

Familia Cirrhitidae

[C] [2] Cirrhitichtys oxycephalus (Bleeker, 1855) [IP] [BT, OO, *]

[1] Cirrhitus rivulatus Valenciennes, 1855 [EP] [BT, OO, *]

Oxycirrhites typus Bleeker, 1857 [IP] [OO, *]

Familia Mugilidae

Mugil cephalus Linnaeus, $1758[\mathrm{CT}][\mathrm{BT}, *]$

[E] Mugil curema Valenciennes, $1836[\mathrm{ANF}][*]$

Familia Labridae

[C] [3] Bodianus diplotaenia (Gill, 1863) [EP] [BT, OO, *]

Decodon melasma Gomon, 1974 [EP] [*]

[E] [1] Halichoeres chierchiae Caporiacco, 1947 [EP] [BT, OO, *]

[E] [1] Halichoeres dispilus (Gunther, 1864) [EP] [*]

Halichoeres melanotis (Gilbert, 1890) [EP] [*]

[E] [1] Halichoeres nicholsi (Jordan y Gilbert, 1881) [EP] [OO, *]

Halichoeres notospilus (Gunther, 1864) [EP] [*]

Halichoeres semicinctus (Ayres, 1859) [EP] [*]

Hemipteronotus mundiceps (Gill, 1863) [END] [OO]

Novaculichthys taeniourus (Lacepede, 1801) [IP] [OO, *]

Pseudojulis inornatus Gilbert, 1890 [END] [OO, *]

[A] [3] Thalassoma grammaticum Gilbert, 1890 [EP] [BT, OO, *]

[A] [3] Thalassoma lucasanum (Gill, 1863) [EP] [BT, DC, OO, *] Xyrichthys pavo (Valenciennes, 1840) [IP] [*]

Familia Scaridae

Nicholsina denticulata (Evermann y Radeliffe, 1917) [EP] [OO, *]

[E] [1] Scarus compressus (Osburn y Nichols, 1916) [EP] [BT, OO, *]

[E] [1] Scarus ghobban Forskaal, 1775 [IP] [BT, OO, *]

[E] Scarus perrico Jordan y Gilbert, 1881 [EP] [BT, HQ, OO, *]

[E] [2] Scarus rubroviolaceus Bleeker, 1847 [IP] [BT, OO, *]

Familia Opistognathidae

Opistognathus scops (Jenkins y Evermann, 1889) [EP] [OO]

Familia Tripterygiidae

Axoclinus carminalis (Jordan y Gilbert, 1882) [EP] [BT, OO]

Enneanectes reticulatus Allen y Robertson, 1991 [END] [OO] 
Familia Dactyloscopidae

Dactyloscopus byersi Dawson, 1969 [EP] [OO]

Dactyloscopus lunaticus Gilbert, 1890 [EP] [OO]

Dactyloscopus mundus Gill, 1862 [EP] [BT, OO]

Dactyloscopus pectoralis Gill, 1861 [EP] [OO]

Gillelus arencola Gilbert, 1890 [EP] [OO]

Gillelus ornatus Gilbert, 1896 [END] [DC, OO]

Gillelus semicinctus Gilbert, 1890 [EP] [OO]

Heteristius cinctus (Osburn y Nichols, 1916 [EP] [OO]

Myxodagnus opercularis Gill, 1861 [EP] [OO]

Familia Labrisomidae

Labrisomus multiporosus Hubbs, 1953 [EP] [BT, OO]

Labrisomus striatus Hubbs, 1953 [EP] [BT, DC, OO]

Labrisomus xanti Gill, 1860 [EP] [BT, OO]

Malacoctenus hubbsi Springer, 1959 [EP] [BT, OO]

Malacoctenus margaritae (Fowler, 1944) [EP] [BT, OO]

Malacoctenus tetranemus (Cope, 1877) [EP] [BT, OO]

Malacoctenus zacae Springer, 1959 [EP] [OO]

Paraclinus beebei Hubbs, 1952 [EP] [OO]

Paraclinus mexicanus (Gilbert, 1904) [EP] [BT, OO]

Paraclinus sini Hubbs, 1952 [EP] [BT, OO]

Paraclinus tanygnathus Rosenblatt y Parr, 1969 [EP] [OO]

Starksia spinipenis (Al-Uthman, 1960) [EP] [BT, OO]

Xenomedea rhodopyga Rosenblatt y Taylor, 1971 [EP] [BT, OO]

Familia Chaenopsidae

Acanthemblemaria crockeri Beebe y Tee-Van, 1938 [END] [BT, OO]

Acanthemblemaria macrospilus Brock, 1940 [EP] [BT, OO]

Chaenopsis alepidota (Gilbert, 1890) [EP] [OO]

Chaenopsis coheni Bohlke, 1957 [END] [OO]

Coralliozetus angelica (Bohlke y Mead, 1957) [EP] [BT, OO]

Coralliozetus micropes (Beebe y Tee-Van, 1938) [EP] [BT, OO]

Coralliozetus rosenblatti Stephens, 1963 [EP] [OO]

Ekemblemaria myersi Stephens, 1963 [EP] [OO]

Emblemaria hypacanthus (Jenkins y Evermann, 1889) [END] [OO]

Emblemaria walkeri Stephens, 1963 [END] [TH, OO]

Protemblemaria bicirris (Hildebrand, 1946) [EP] [OO]

Protemblemaria lucasana Stephens, 1963 [END] [OO]

Stathmonotus sinuscalifornici (Chabanaud, 1942) [END] [OO]

Familia Blenniidae

Entomacradus chiostictus (Jordan y Gilbert, 1883) [EP] [BT, *]

Hypsoblennius brevipinnis (Gunther, 1861) [EP] [BT, OO]

Hypsoblennius gentilis (Girard, 1854) [EP] [DC]

[C] [3] Ophioblennius steindachneri Jordan y Evermann, 1898 [EP] [BT, OO, *]

[C] [1] Plagiotremus azaleus (Jordan y Bollman, 1890) [EP] [BT, OO, *]

Familia Gobiidae

Aruma histrio (Jordan, 1884) [END] [TH, DC, OO]

Barbulifer mexicanus Hoese y Larson, 1985 [END] [OO]

Barbulifer pantherinus (Pellegrin, 1901) [END] [OO]

Bathygobius ramosus Ginsburg, 1947 [EP] [BT, OO]

Chriolepis zebra Ginsburg, 1938 [END] [BT, OO]

[E] Coryphopterus urospilus Ginsburg, 1938 [EP] [OO, *]

Elacatinus digueti (Pellegrin, 1901) [EP] [OO]

Elacatinus puncticulatus (Ginsburg, 1938) [EP] [BT, OO, *]

Gobiosoma chiquita (Jenkins y Evermann, 1889) [END] [DC, OO]

Gobiosoma nudum (Meek y Hildebrand, 1928) [ANF] [BT, TH, OO]

Gobulus crescentalis (Gilbert, 1892) [EP] [TH, OO]

Gymneleotris seminudus (Gunther, 1864) [EP] [BT, OO]

Lythrypnus dalli (Gilbert, 1890) [EP] [OO]

Lythrypnus pulchellus Ginsburg, 1939 [EP] [OO] 
Familia Acanthuridae

[E] [1] Acanthurus nigricans (Linnaeus, 1758) [IP] [*]

[E] Acanthurus triostegus (Linnaeus, 1758) [IP] [DC, OO, *]

Acanthurus xanthopterus Valenciennes, 1835 [IP] [OO,
[C] [3] Prionurus punctactus Gill, 1862 [EP] [BT, TH, OO, *]

Familia Zanclidae

[E] Zanclus canescens (Linnaeus, 1758) [IP] [BT, DC, OO, *]

Familia Scombridae

Euthynnus lineatus Kishinouye, 1920 [EP] [DC]

Katsuwonus pelamis (Linnaeus, 1758) [CT] [SR]

Thunnus albacares (Bonnaterre, 1788) [CT] [HQ]

Orden Pleuronectiformes

Familia Bothidae

Citharichthys gordae Beebe y Tee-Van, 1938 [END] [OO]

Paralichthys woolmani Jordan y Williams en Gilbert, 1897 [EP] [OO]

Orden Tetraodontiformes

Familia Balistidae

[E] Balistes polylepis Steindachner, $1876[\mathrm{EP}]\left[{ }^{*}\right]$

[E] Melichthys niger (Bloch, 1786) [CT] [*]

[1] Pseudobalistes naufragium (Jordan y Starks, 1895) [EP] [*]

[C] [3] Sufflamen verres (Gilbert y Starks, 1904) [EP] [BT, DC, OO, *]

Familia Monacanthidae

Aluterus scriptus (Osbeck, 1765) [CT] [*]

Cantherinus dumerilii (Hollard, 1854) [IP] [TH, *]

Familia Ostracidae

Ostracion meleagris Shaw, 1796 [IP] [*]

Familia Tetraodontidae Arothron hispidus (Linnaeus, 1758) [IP] [OO]

[E] [2] Arothron meleagris (Bloch y Schneider, 1801) [IP] [BT, DC, OO, *]

[E] [3] Canthigaster punctatissima (Gunther, 1870) [EP] [BT, OO, *]

Sphoeroides annulatus (Jenyns, 1842) [EP] [OO, *]

Sphoeroides lobatus (Steindachner, 1870) [EP] [OO]

Familia Diodontidae

Diodon holocanthus Linnaeus, 1758 [CT] [BT, OO, *]

[E] [1] Diodon hystrix Linnaeus, 1758 [CT] [BT, OO, *]

Clave para la abundancia relativa: A: Abundante (más del 5\% de la abundancia total observada entre 1986 y 1997); C: Común (entre el $1 \%$ y el 5\%); E: Escasa (del 0.1 al 1\%). Clave para la frecuencia relativa: 3: (la especie apareció en el $75 \%$ al 100\% de los censos); 2: (vista en el 50\% al 75\% de los censos); 1: (del 25\% al 50\%). Clave para la afinidad biogeográfica: PAN Especie panámica (se distribuye exclusivamente en la Provincia Panámica); COS: Especie cosmopolita; IP: Especie del Indo Pacífico; ANF: Especie anfiamericana (se distribuye en costas occidentales y orientales del Continente Americano); CT: Especie circuntropical; END: Especie endémica del Golfo de California. Registros previos en la localidad: SR: Steinbeck \& Ricketts 1941; BT: Brusca \& Thomson 1975; HQ: Holguín-Quiñones 1976; TH: Thomson et al. 1979; M: Montgomery 1980a, b; DC: de la Cruz-Agüero 1997; OO: Observaciones de otros investigadores (R.H. Rosenblatt y J. de la Cruz-Agüero); *: Observaciones en campo (entre 1986 y 1998).

Key for relative abundance: A: Abundant (more than 5\% of total abundance observed from 1986 to 1998); C: Common (between 1\% and 5\%); E: Scarce (from $0.1 \%$ to $1 \%$ ). Key for relative frequency: 3: (the species appeared from $75 \%$ to $100 \%$ of census); 2: (seen from $50 \%$ to $75 \%$ of census); 1 : (from $25 \%$ to $50 \%$ ). Key for biogeographic affinity: PAN: Panamic species (exclusively distributed in the Panamic Province); COS: Cosmopolitan species; IP: Indo-Pacific species; ANF: Amphiamerican species (distributed in eastern and western coasts of the American Continent); CT: Circumtropical species; END: Gulf of California endemic species. Previous records in the locality: SR: Steinbeck \& Ricketts 1941; BT: Brusca \& Thomson 1975; HQ: Holguín-Quiñones 1976; TH: Thomson et al. 1979; M: Montgomery 1980a, b; DC: de la Cruz-Agüero 1997; OO: Observations of other researchers (R.H. Rosenblatt and J. de la Cruz-Agüero); *: Field observations (from 1986 to 1998. 


\section{DISCUSIÓN}

Comparando con la única recopilación previa sobre la fauna ictiológica de Cabo Pulmo (Brusca \& Thomson 1975) el número de táxones registrados se duplicó con el presente trabajo, elevándose de 108 a 236 (Cuadro 1). La diferencia se debe particularmente a que en el trabajo de 1975 casi no se incluyeron especies de algunas familias como Ophichthidae, Serranidae, Labridae, Dactyloscopidae, Chaenopsidae o Gobiidae, las que representan un alto porcentaje de la fauna total en el arrecife.

La riqueza de especies encontrada en el área de estudio (Cuadro 1) es notablemente alta. En Cabo Pulmo se encontraron 178 de las 271 especies de zonas rocosas que habitan el golfo $(65.1 \%)$, además de un $35 \%$ de todas aquellas que ocupan fondos someros en la región (Thomson \& Gilligan 1983). Comparando con la fauna ictiológica de otros arrecifes rocosos o coralinos del Pacífico mexicano situados en la Bahía de $\mathrm{La} \mathrm{Paz}\left(24^{\circ} \mathrm{N}, 116\right.$ especies; Pérez-España et al. 1996, Sánchez-Ortíz et al. 1997), Isla Cerralvo ( $24^{\circ} \mathrm{N}, 174$ especies; Galván-Magaña et al. 1996), las Islas Revillagigedo $\left(18^{\circ} \mathrm{N}, 187\right.$ especies; Allen \& Robertson 1994, Fischer et al. (1995), Castro-Aguirre \& Balart en prensa), la costa de Colima $\left(18^{\circ} \mathrm{N}\right.$, 203 especies; Pérez-Vivar 1995) o incluso con el Atolón Clipperton ( $10^{\circ} \mathrm{N}, 132$ spp.; Allen \& Robertson 1997), puede verse que en Cabo Pulmo se llega a exceder las cifras registradas de riqueza íctica, lo cual es relevante considerando que éste arrecife cubre un área mucho más pequeña que las demás. Todo lo anterior remarca la importancia ictiológica de Cabo Pulmo, no sólo en el Golfo de California sino a nivel de todo el Pacífico mexicano.

En relación a la abundancia, de las 36 especies dominantes (importantes tanto en abundancia como en frecuencia de registro), 17 son carnívoras o zooplanctívoras, 11 omnívoras y ocho herbívoras (con base en la clasificación de tipos de presas aparecida en Thomson \& Gilligan 1983, y Allen \& Robertson 1997), lo que sugiere que muchos de los peces importantes del arrecife de Cabo Pulmo tienen amplios espectros alimenticios. Tanto el balance en la proporción de tipos de dieta como la alta riqueza específica de peces en Cabo Pulmo pueden ser consecuencia de que en la zona hay muy variados microambientes, como la zona coralina principal, el borde arrecifal, cuevas y paredes de pendiente abrupta, o parches arenosos (Squires 1959, Brusca \& Thomson 1975, Reyes-Bonilla 1993), cada uno con características propias y ocupado por presas de muy distintos grupos taxonómicos. Tal variedad de hábitats y nichos ha permitido que en una zona geográficamente muy limitada pueda convivir un gran número de especies y con ello, ha dado lugar a que actualmente este arrecife deba ser considerado como una de las localidades más importantes de la región, bajo la óptica de la fauna ictiológica.

\section{AGRADECIMIENTOS}

Este estudio estuvo apoyado por la UABCS, DGICSA-SEP, el Centro de Investigación Científica y Educación Superior de Ensenada y el Fondo Mexicano para la Conservación de la Naturaleza. Sin embargo, no hubiera sido posible su realización sin la ayuda de los residentes de la Bahía de Cabo Pulmo, en particular los miembros de las familias Castro y Mijares. Saúl Rodríguez, Juan Gabriel Díaz, Almei Moehl, Gabriela Anaya, Jessica Cifuentes, Víctor Hugo Beltrán y Edgardo Ochoa, entre otros (UABCS), contribuyeron en las labores de censos. Varios investigadores proporcionaron datos para esta recopilación, pero en particular se agradece la colaboración de Richard H. Rosenblatt (Scripps Institution of Oceanography, San Diego, E.U.A.) y José de la Cruz Aguiero (CICIMAR- IPN, La Paz). El manuscrito fue revisado por Gustavo Danemann (PRONATURA, Ensenada), Carlos Villavicencio (UABCS), Horacio Pérez (CICIMAR-IPN, La Paz) y Eduardo Balart (CIBNOR, La Paz), y por tres árbitros anónimos. 


\section{REFERENCIAS}

Allen, G.R. \& D.R. Robertson. 1994. Fishes of the tropical eastern Pacific. Univ. of Hawaii, Honolulu. 332 p.

Allen, G.R. \& D.R. Robertson. 1997. An annotated checklist of the fishes of Clipperton atoll, tropical eastern Pacific. Rev. Biol. Trop. 45: 813-843.

Anaya-Reyna, G. \& R. Riosmena-Rodríguez. 1996. Macroalgas del arrecife coralino de Cabo Pulmo- Los Frailes, B.C.S., México. Rev. Biol. Trop. 44: 903906.

Baynes, T.W. 1999. Factors structuring a subtidal encrusting community in the southern Gulf of California. Bull. Mar. Sci. 64: 419-450.

Bohnsack, J.A. \& S.P. Bannerot. 1986. A stationary visual census technique for quantitatively assesing community structure of coral reef fishes. NOAA Techn. Rep. NMFS 41: 1-15.

Brusca, R.C. \& D.A. Thomson. 1975. Pulmo reef: the only "coral reef" in the Gulf of California. Ciencias Marinas 1: 37-53.

Castro-Aguirre, J.L. \& E. Balart. En prensa. La ictiofauna de las Islas Revillagigedo y sus relaciones zoogeográficas, con comentarios acerca de su origen y evolución. In L. Medrano-González, O. Holguín-Quiñones y A. Ortega-Rubio (eds.). Las Islas Revillagigedo, México. Avances en la investigación y propuestas de conservación. Universidad Nacional Autónoma de México/ Centro Interdisciplinario de Ciencias Marinas/ Centro de Investigaciones Biológicas del Noroeste, México, D.F.

Cintra-Buenrostro, C.E., H. Reyes-Bonilla \& O. ArizpeCovarrubias. 1999. Los equinodermos (Echinodermata) del arrecife de Cabo Pulmo, Pacífico de México. Rev. Biol. Trop. 46: 341: 344.

De la Cruz-Agüero, J. 1997. Catálogo de los peces marinos de Baja California Sur. Centro Interdisciplinario de Ciencias Marinas/ Comisión Nacional para el Conocimiento y Uso de la Biodiversidad, La Paz. 346 p.

Fischer, W., F. Krupp, W. Schneider, C. Sommer, K.E. Carpenter \& V.H. Niem. 1995 (eds.). p. 648-1652. In Guía FAO para la identificación de especies para los fines de la pesca. Pacífico centro oriental. Vol. II y III. Vertebrados (Partes 1 y 2 ). FAO, Roma.
Galván-Magaña, F., L.A. Abitia-Cárdenas, J. RodríguezRomero, H. Pérez-España \& H. Chávez-Ramos. 1996. Lista sistemática de los peces de la Isla Cerralvo, Baja California Sur, México. Ciencias Marinas 22: 295-311.

García-Madrigal, M.S. En prensa. Anomuros (Anomura) del arrecife de Cabo Pulmo, Golfo de California, México. Rev. Biol. Trop. 47(2):

García-Madrigal, M.S. \& J.R. Bastida-Zavala. En prensa. Cangrejos braquiuros (Brachyura) del arrecife de $\mathrm{Ca}$ bo Pulmo, Golfo de California, México. Rev. Biol. Trop. 47: 119-128.

Holguín-Quiñones, O.E. 1976. Catálogo de especies marinas de importancia comercial en Baja California Sur. Instituto Nacional de la Pesca/ Estación de Investigación Pesquera de La Paz, La Paz. 117 p.

Montgomery, M.L. 1980a. The impact of non- selective grazing by the giant blue damselfish Miscrospathodon dorsalis on algal communities in the Gulf of California, Mexico. Bull. Mar. Sci. 30: 290-303.

Montgomery, M.L. 1980b. Comparative feeding ecology of two damselfishes (Pomacentridae: Teleostei) from the Gulf of California. J. Exp. Mar. Biol. Ecol. 47: 9-24.

Nelson, J.S. 1994. Fishes of the World. $2^{\circ}$ ed. Wiley, Nueva York. 416 p.

Pérez-España, H., F. Galván-Magaña \& L.A. Abitia-Cárdenas. 1996. Variaciones temporales y espaciales en la estructura de la comunidad de peces de arrecifes rocosos del suroeste del Golfo de California, México. Ciencias Marinas 22: 273-294.

Pérez-Vivar, T.L. 1995. Sistemática y biogeografía de peces del litoral de Colima, México. Tesis de Licenciatura, Universidad de Guadalajara, Guadalajara, México.

Reyes-Bonilla, H. 1997. Cabo Pulmo reef: a new marine reserve in the Gulf of California. Conserv. Biol. 11: 827.

Reyes-Bonilla, H., F. Sinsel-Duarte \& O. Arizpe-Covarrubias. 1997. Gorgonias y corales pétreos (Anthozoa: Gorgonacea y Scleractinia) del arrecife de $\mathrm{Ca}$ bo Pulmo, Pacífico de México. Rev. Biol. Trop. 45: 1439-1443. 
Sale, P.F. 1997. Visual census of fishes: how well do we see what is there? Proc. $8^{\text {th }}$ Int. Coral Reef Symp., Panamá 2: 1435-1440.

Sánchez-Ortíz, C., J.L. Arreola-Robles, O. Aburto-Oropeza \& M. Cortés-Hernández. 1997. Peces de arrecife en la región de La Paz, B.C.S., p. 177-188. In J. Urbán-Ramírez \& M. Ramírez-Rodríguez (eds.). La Bahía de La Paz. Investigación y conservación. Universidad Autónoma de Baja California Sur/ Centro Interdisciplinario de Ciencias Marinas/ Birch Aquarium, La Paz.

Squires, D.F. 1959. Corals and coral reefs in the Gulf of California. Bull. Amer. Mus. Nat. Hist. 118: 370-431.
Steinbeck, J. \& E.F. Ricketts. 1941. Sea of Cortez. Viking, Nueva York. 598 p.

Thomson, D.A. \& M. Gilligan. 1983. The rocky shore fishes, p. 98-129. In T.J. Case \& M.L. Cody (eds.). Island biogeography in the Sea of Cortez. University of California, Berkeley.

Thomson, D.A., L.T. Findley \& A.N. Kerstitch. 1979. Reef fishes of the Sea of Cortez. Wiley, Nueva York. 302 p.

Villarreal-Cavazos, A. 1988. Distribución y diversidad de peces en el arrecife coralino de Cabo PulmoLos Frailes, B.C.S. Tesis de Licenciatura. Universidad Autónoma de Baja California Sur. La Paz. 